\title{
Analysis and Characterization of a High Performance Ka-band Surface Micromachined Elevated Patch Antenna
}

\author{
Bo Pan, Yong-Kyu Yoon, Member, IEEE, George E. Ponchak, Senior Member, IEEE, Mark G. Allen, Senior Member, \\ IEEE, John Papapolymerou, Senior Member, IEEE and Manos M. Tentzeris, Senior Member, IEEE
}

\begin{abstract}
A novel Ka-band surface micromachined air-elevated patch antenna is designed, fabricated and characterized. It demonstrates a $10.5 \% \quad 10-\mathrm{dB}$ fractional bandwidth and $9.5 \mathrm{dBi}$ directivity for an elevation of $600 \mu \mathrm{m}$. The elevated patch is fed by a vertical epoxy-core metal-coated micromachined probe and supported by several epoxy posts underneath. By the unique feeding technique and the substrate removal approach that are presented in this paper, the performance degradation for traditional microstrip antenna directly built on high- $\varepsilon_{\mathrm{r}}$ substrate can be significantly alleviated.

Index Terms - air-elevated antenna, surface micromachining, antenna feeding, on-wafer radiation pattern measurement
\end{abstract}

\section{INTRODUCTION}

$\mathrm{W}$ ITH THE ever-increasing demand for high performance, low cost and low-power wireless communication systems, the need for integrating all functional modules on a single chip or into a single package becomes more pronounced [1][2]. There have been various efforts to fully integrate numerous antenna geometries onto MMIC-compatible wafers. Microstrip antennas are widely used as it is a good candidate for on-wafer integration except that it has different substrate requirements in terms of $\varepsilon_{\mathrm{r}}$ and thickness from the rest of the integrated functions and circuits. More specifically, for the rest of the RF components/passives, high- $\varepsilon_{\mathrm{r}}$ and thin materials are commonly used for the sake of compactness and thermal dissipation. However, microstrip antenna designs prefer low- $\varepsilon_{\mathrm{r}}$ and thick substrates to suppress the substrate modes (higher efficiency), achieve a wider bandwidth and undisturbed radiation patterns, and reduce any undesired coupling between the various elements in array configurations.

Many efforts have been put forth to alleviate the problem caused by surface waves. Among them, [3] uses lateral etching of silicon underneath the patch to lower the effective dielectric constant. [4] uses metal posts to lift the patch into air and use a proximity coupling method to feed the patch. For both [3] and [4], the effective substrate of the patch antenna is a mixture of air and high- $\varepsilon_{\mathrm{r}}$ materials, so compared to the completely air filled elevated patch shown in this paper, the antenna performance is not fully optimized. It has been demonstrated in [5] that the fabrication of a 200 micron elevated $60 \mathrm{GHz} 2$ $\mathrm{x} 1$ patch array supported by three metal posts is feasible without showing radiation pattern data. Since there are several metal pins connecting the ground and the patch in the area around the middle of patch, only half of the patch radiates.

This letter will present the design and full characterization of a new substrate-independent elevated microstrip patch antenna using the feeding probe [6] that was also used by the authors of [5]. The very preliminary result of this research was first reported in [7], only demonstrating the idea. This antenna is completely lifted into the air and radiates around $27 \mathrm{GHz}$.

\section{ANTENNA ANALYSIS AND FABRICATION}

\section{A. Motivation of elevating}

The proposed structure is shown in Fig. 1 with the design parameters summarized in Table 1. A number of polymer posts are supporting the elevated antenna and one CPW-fed vertical probe [5][6] is used to feed it, offering several advantages. First, this topology effectively creates a low- $\varepsilon_{\mathrm{r}}$ substrate since the substrate is essentially air (the lowest possible dielectric constant). This will help increase the radiation efficiency, directivity, and the radiation bandwidth. In addition, for traditional patch antennas integrated with other components, once the substrate specifications are chosen for the whole module, the thickness is a universal variable for each component on this substrate and it cannot be tailored freely and thus optimized for antenna design. On the contrary, for the air-elevated patch antenna presented in the paper, the height of the vertical probe and polymer posts can be specified in the process and vary from several microns up to $1 \mathrm{~mm}$, regardless of substrate used. This can be used to further enhance the patch bandwidth when we cannot change the substrate thickness for the sake of the rest of the module circuits. Thus, the air-elevated patch can meet different application requirements and be utilized as a substrate-independent solution. Last, but not least, the (CPW) ground on top of the wafer will totally shield the antenna from the underlying elements and vice versa.

\section{B. Impact of vertical feeding probe}

Several parametric analyses for various geometrical parameters have been performed and among these parameters, the current probe radius plays a very important role. Since the 
probe is implemented by the epoxy-core metal-coated technique [7] [8], it does not have to use the standardized center pin diameter of the traditional coaxial connector. Its diameter can be optimized to get optimal performance.

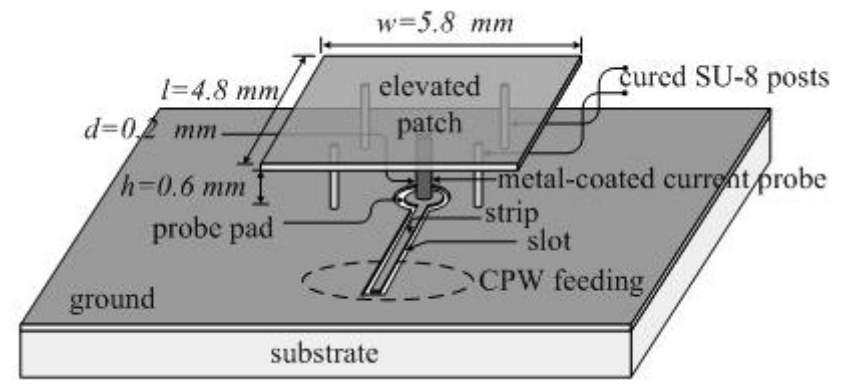

Fig 1 Proposed CPW-fed elevated patch antenna

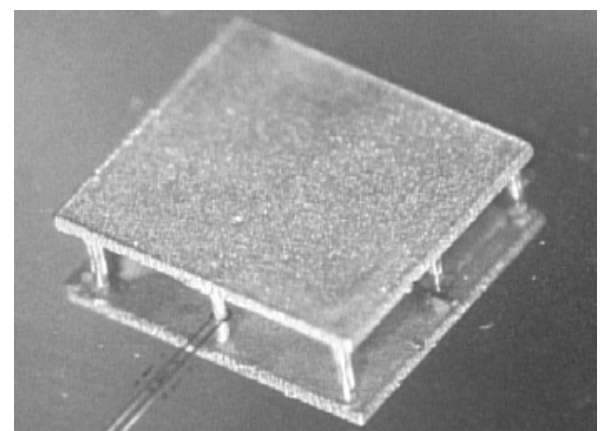

Fig 2 Picture of fabricated post-supported patch antenna

In this design, the reference plane to calculate the input impedance of the proposed structure is set right before the $\mathrm{CPW}$-vertical probe transition, thus the imaginary part of the $Z_{\text {in }}$ comes from the antenna itself, as well as the feeding probe, that can be modeled as a series inductor in the equivalent circuit. Various values of the diameter of the probe have been simulated and compared using Ansoft HFSS. Figure 3 shows the $\mathrm{S}$ parameters locus when the other geometric parameters are as specified in Table 1. When increasing the probe diameter from $100 \mu \mathrm{m}$ to $300 \mu \mathrm{m}$, wire inductance associated with the probe decreases. Thus for the $Z_{i n}$, the slope of the reactance becomes less steep. Consequently, more frequency points will fall within the $\mathrm{SWR}=2$ circle and more bandwidth can be achieved. On the other hand, increasing the probe diameter will enlarge the probe pad and thus the perimeter of the circular slot around the probe pad; at the same time, the CPW line signal/slot widths also need to be increased to achieve smooth impedance transition to the probe. This introduces more ground opening and disturbs the radiation pattern. (As will be observed in Sec . III). Because of this finding, $200 \mu \mathrm{m}$ probe diameter is chosen.

Like other classical probe-fed antennas, the probe location was chosen as $1 \mathrm{~mm}$ away from the patch front edge to make the real part of the input impedance close to $50 \mathrm{Ohm}$.

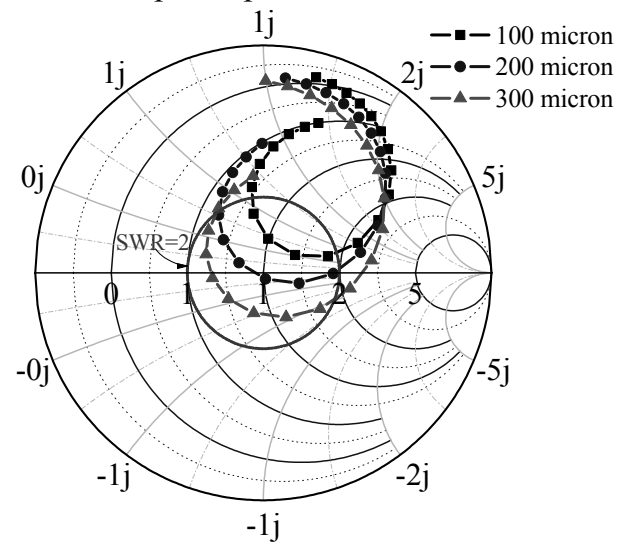

Fig 3 Matching locus when varying diameter of the feeding probe

\section{Impact of epoxy posts}

Simulated data for the geometries with and without epoxy $\left(\varepsilon_{\mathrm{r}}=3\right)$ posts demonstrate no significant difference in terms of bandwidth, radiation pattern and electrical/magnetic field distribution, as it is evident from Figure 4 that shows the surface current distribution on the patch. The number of posts and their volumes should be determined considering the tradeoff between electrical performance and mechanical robustness. A large number of posts would offer a stronger and more rigid mechanical performance at the expense of an increased dielectric loading of the patch, and thus of a higher dielectric loss.

In this design, the smallest possible number of posts, while maintaining mechanical robustness, is decided as eight; one polymer supporting post is used for each patch vertex and one for each middle point of the patch edge. Since the current probe is very close to the front edge, the one at that edge has been removed. One polymer post is also placed in the center of patch. These eight polymer posts account for $0.8 \%$ of the cavity volume underneath the patch. Thus, the loss and the dielectric loading of the supporting posts only play a minor role and can be omitted. The photograph of the patch in Figure 2 shows the location of 4 of the supporting posts and the current feed.

Mechanical analysis was also performed to evaluate the mechanical properties of the proposed geometry. Simulation results by the FEM-based mechanical simulation tool ABAQUS ${ }^{(\mathrm{TM})}$ indicate that the structure is both mechanically and electically robust under vertical pressure, but fragile to shear stress. Thus operation under the latter circumstance should be avoided.

TABLE I

DESIGN PARAMETERS FOR PROPOSED STRUCTURE

\begin{tabular}{c|cccccccc}
\hline & $\begin{array}{c}\text { patch } \\
\text { length } l\end{array}$ & $\begin{array}{c}\text { patch } \\
\text { width } w\end{array}$ & $\begin{array}{c}\text { patch } \\
\text { thickness } t\end{array}$ & $\begin{array}{c}\text { patch } \\
\text { elevation } h\end{array}$ & $\begin{array}{c}\text { substrate } \\
\varepsilon_{\mathrm{r}}\end{array}$ & $\begin{array}{c}\text { substrate } \\
\text { thickness }\end{array}$ & $\begin{array}{c}\text { probe } \\
\text { location } \\
\text { off edge }\end{array}$ & $\begin{array}{c}\text { probe } \\
\text { diameter } d\end{array}$ \\
\hline value & $4.8 \mathrm{~mm}$ & $5.8 \mathrm{~mm}$ & $0.1 \mathrm{~mm}$ & $0.6 \mathrm{~mm}$ & 8.1 & $0.6 \mathrm{~mm}$ & $1 \mathrm{~mm}$ & $200 \mu \mathrm{m}$ \\
\hline
\end{tabular}




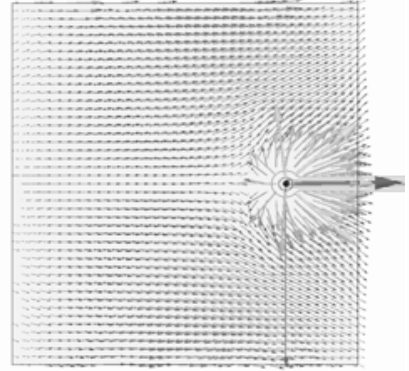

a)

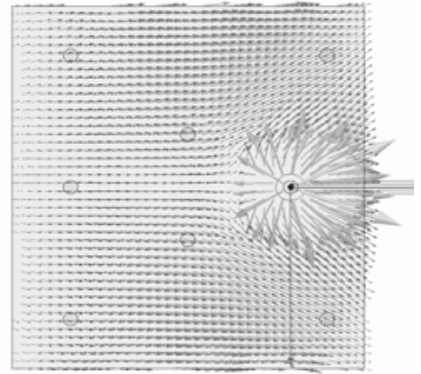

b)
Fig. 4 Current distribution on patch a)without and b)with polymer posts

\section{Fabrication of the structure by advanced MEMS technologies}

The proposed polymer-post supported patch antenna discussed above was fabricated on top of $0.6-\mathrm{mm}$ thick soda-lime glass. The polymer core of metalized feeding probe was defined with $600 \mu \mathrm{m}$ thick SU-8 (MicroChem SU-8 2100). A conformal seeding layer of $\mathrm{Ti} / \mathrm{Cu} / \mathrm{Ti}$ was deposited using a DC sputterer. Negative-tone photoresist NR-9 8000 was spun on top of the Ti layer and patterned to cover the slot of the feeding CPW-line and copper was electro-plated to metalize the current probe and ground pattern. $600 \mu \mathrm{m}$ thick SU-8 was dispensed and patterned for a second time to define the supporting polymer posts.

After all posts had been formed, the assembly of the top patch with the supporting structure was done by flipping the whole structure to align with the vacuum-stuck patch on the bench. The relative positioning was realized with the aid of marker on the ground plane. Silver paste was pre-applied to the post contact areas on the patch using a tiny tip to guarantee good electrical contact between the current probe and the patch. The whole structure was then treated by electroplating bonding techniques detailed in [9]. At this stage, NR9-8000 was removed and $\mathrm{Ti} / \mathrm{Cu} / \mathrm{Ti}$ in the slot region was etched. The structure was then released. In the future, batch processing of the elevated patch antenna may be performed using flip chip processing with predefined bumps for positioning to the posts.

\section{CHARACTERIZATION AND DISCUSSIONS}

\section{A. Return loss}

The measured return loss of the elevated microstrip patch antenna, with the parameters given in Table. 1, is plotted along with the simulation results in Figure 5. A 10.5 percent $10-\mathrm{dB}$ bandwidth (26.2 GHz $29.1 \mathrm{GHz})$ is observed in the measurements. This bandwidth is slightly higher than the simulated one, which has a 9.6 percent bandwidth. This is believed to come from various losses that cannot be accurately modeled in numerical simulations; these losses will pull down the reflection coefficient curve in measurement. The central frequency is shifted up by $2 \%$ from $27.2 \mathrm{GHz}$ to $27.7 \mathrm{GHz}$, which can be attributed to accumulated fabrication dimension variations and tolerances.

As a comparison, the traditional patch antenna directly printed on top of this kind of soda-lime glass (600 micron thick, $\varepsilon_{\mathrm{r}}=8.1$ ) gives a narrower fractional bandwidth of $2.6 \%$ according to simulations. It is also very lossy.

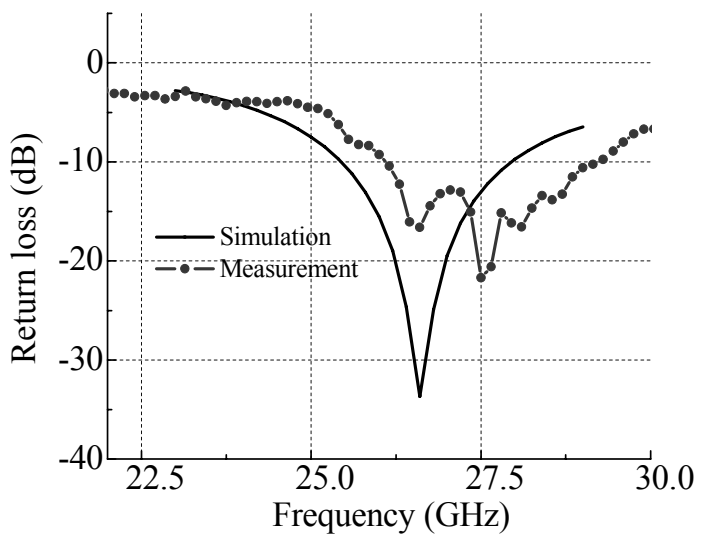

Fig. 5 Simulation and measurement of the return loss for the elevated patch antenna

\section{B. Radiation pattern}

The radiation pattern is characterized with an on-wafer radiation pattern probing system that has been modified from the original system [10]. In the new system, the microwave probe station is replaced by a plexiglass pedestal that holds a microwave probe head and a $3.8 \mathrm{~cm}$ thick Styrofoam cantilever upon which the antenna sets. Furthermore, a modified GGB Industries Picoprobe with a microcoax length of $2.54 \mathrm{~cm}$ is used to reduce the interaction of the probe head with the antenna under test. With this new on-wafer radiation pattern measurement system, the only metal structure near the antenna is the probe head, which after being covered by a piece of absorber, results in smooth radiation plots. However, even this small structure prevents the E-plane radiation pattern across the full 180 degree sweep. During the test, the elevated microstrip antenna is the transmitting antenna and a Ka-Band, $15 \mathrm{~dB}$ gain horn is used as the receiving antenna with a separation of $27 \mathrm{~cm}$ between them. Before measurements, the diode power detector is calibrated and all measurements are corrected by this calibration factor and the gain factor of the standard gain horn used during calibration.

The proposed antenna has demonstrated a significantly improved radiation performance than the traditional patch antenna directly printed on top of the same type of soda-lime glass. The comparison is shown in Table 2. The maximum predicted directivity for the elevated patch is $9.5 \mathrm{dBi}$ and the antenna efficiency is $94 \%$. A non-elevated patch on the same substrate gives a maximum predicted directivity of $4.9 \mathrm{dBi}$, but no longer at the broadside direction as a consequence of surface wave triggered in this electrically thick, high- $\varepsilon_{\mathrm{r}}$ substrate. (As in Figure 6) It can be clearly observed from Fig. 6 (b) that there is a deep drop in the z-direction for the antenna without elevation due to the diffraction of surface waves at the edge of the substrate. Elevated patch have a clear main beam in the z-direction.

The E-plane and H-plane patterns at the measured central frequency of $27.7 \mathrm{GHz}$ are plotted in Figure 7 and Figure 8 respectively. The simulation results predicted by Zeland IE3D and Ansoft HFSS are plotted in the same figure for comparing. They agree very well with each other. 


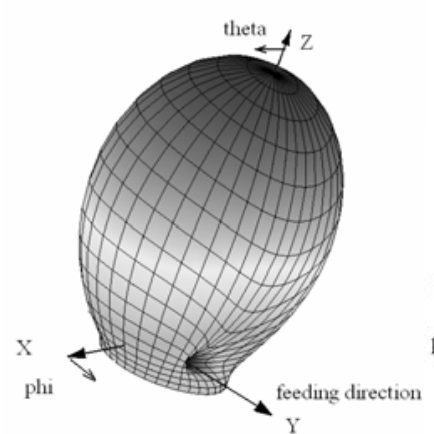

(a)

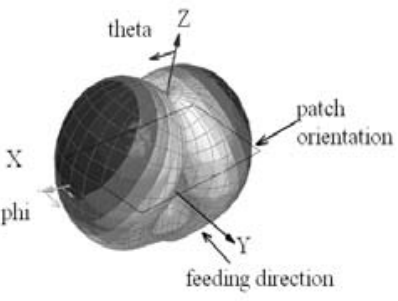

(b)
Fig. 63 -D radiation for the elevated patch (a) and directly printed patch (b)

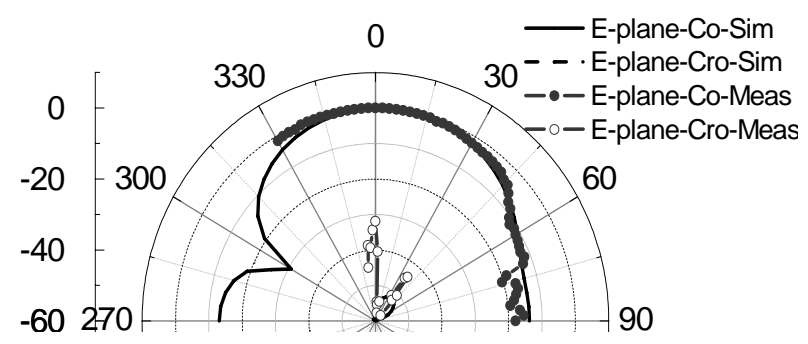

Fig. 7 Simulated and measured E-plane pattern for the elevated patch

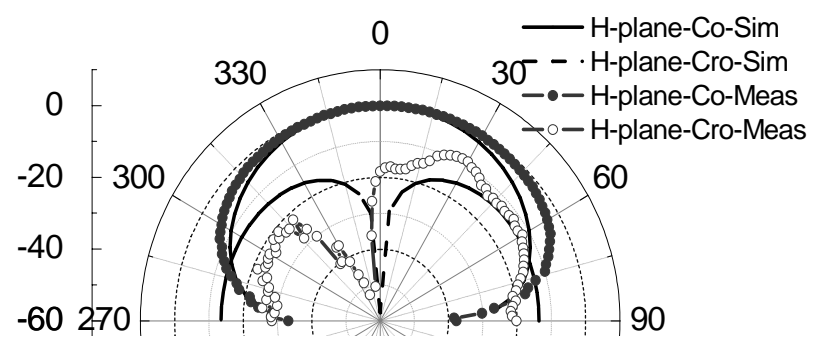

Fig 8 Simulated and measured H-plane pattern for the elevated patch

In the E-plane co-polarization, simulations predict a null in the direction of the feeding CPW-probe transition; this effect is believed to be a consequence of the electromagnetic field perturbation due to the existence of the transition. There is no such phenomenon for traditional probe-fed patch antenna since the ground is continuous and does not have a CPW slot cut.

The maximum simulated and measured E-plane cross-polarization level is $-52 \mathrm{~dB}$ and $-31 \mathrm{~dB}$ respectively. For $\mathrm{H}$-plane, these values are $-13.5 \mathrm{~dB}$ and $-8.8 \mathrm{~dB}$. E-plane cross-polarization level is lower than H-plane because the

\section{TABLE 2}

Comparison of antenna performances with/without elevation

\begin{tabular}{l|cccc}
\hline & $\begin{array}{l}\text { Max } \\
\text { directivity }\end{array}$ & $\begin{array}{l}\text { E-plane } \\
\text { 3-dB beamwidth }\end{array}$ & $\begin{array}{l}\text { H-plane } \\
3-d B \\
\text { beamwidth }\end{array}$ & $\begin{array}{l}\text { Fractional } \\
\text { bandwidth }\end{array}$ \\
\hline $\begin{array}{l}\text { Patch } \\
\text { elevated }\end{array}$ & 9.5 & $75^{\circ}$ & $90^{\circ}$ & $9.6 \%$ \\
\hline $\begin{array}{l}\text { No } \\
\text { elevation }\end{array}$ & 4.9 & N/A & N/A & $2.6 \%$ \\
\hline
\end{tabular}

field yields a null at the broadside direction. Notice that a clear null is observed for the $\mathrm{H}$-plane cross polarization at $\theta=0$ in simulation and the same null shifts slightly to -10 degrees in measurements. A possible reason for the higher level of the cross-polarization level on the right side of the measured diagram could be that the prototype probe is not exactly on the middle line of the patch due to the assembly process.

\section{Conclusions}

In this work, a novel surface micromachined air-elevated patch antenna in Ka-band is reported. It demonstrates increased efficiency and bandwidth and an improved radiation pattern. The fabrication method is compatible with the integration of other RF components on the same substrate, facilitating the application of this antenna in fully integrated SOP-based mm-wave modules.

\section{REFERENCES}

[1] R.R.Tummala, M.Swaminathan, M.M.Tentzeris, et al, 'The SOP for Miniaturized, Mixed-Signal Computing, Communication and COnsumer Systems of the Next Decade", IEEE Trans. on Advanced Packaging, Vol.27 No.2, pp.250-267, May 2004.

[2] M.M.Tentzeris, J.Laskar, J.Papapolymerou, et al, 'RF SoP for Multi-band RF and Millimeter-wave Systems", Advanced Packaging Magazine, pp.15-16, April 2004.

[3] J. Papapolymerou, R.F. Drayton and L.P.B. Katehi, "Micromachined Patch Antennas," IEEE Trans. Antennas and Propagation, Vol. 46, No. 2, pp. 275-283, February 1998..

[4] Man-Lyun Ha, Y. Cho, C. Pyo, and Y. Kwon, "Q-band Micro-patch Antennas implemented on a High Resistivity Silicon substrate using the Surface Micromachining Technology", Proc. of IEEE International Microwave Symposium 2004, pp.1189-1192, June, 2004, Fort Worth, TX.

[5] Jeong-Geun Kim, Hyung Suk Lee, Ho-Seon Lee, et al, "60-GHz CPW-Fed Post-Supported Patch Antenna Using Micromachining Technology", IEEE Microwave and Wireless Components Letters, Vol. 15, No. 10, pp635-637 Oct 2005

[6] B. Pan, Y. Yoon, P. Kirby, et al, "A W-band Surface Micromachined Monopole for Low-cost Wireless Communication Systems," Proc. of IEEE International Microwave Symposium 2004, pp.1935-1938, June 2004, Fort Worth, TX.

[7] B.Pan, Y.Yoon, J.Papapolymerou, M.M.Tentzeris and M.G.Allen, "A High Performance Surface-Micromachined Elevated Patch Antenna", Procs. of the 2005 IEEE-APS Symposium, pp.397-400, vol.1B, Washington, DC, July 2005.

[8] Y.-K Yoon, J.-W. Park, and M.G. Allen, "Polymer-Core Conductor Approaches for RF MEMS," IEEE Journal of Microelectromechanical Systems (MEMS), vol. 14, no. 5, 2005, pp. 886-894

[9] Yeun-Ho Joung, "Electroplating bonding technology for chip interconnect, wafer level packaging and interconnect layer structures," Georgia Institute of Technology, PhD dissertation, 2003.

[10] R. N. Simons, "Novel on-wafer radiation pattern measurement technique for MEMS actuator based reconfigurable patch antennas," 24th Annual Antenna Measurement Techniques Association Meeting and Symposium, Cleveland, OH, Nov. 3-8, 2002 (Also NASA TM 2002-211816)

[11] J. Huang, "The finite ground plane effect on the microstrip antenna radiation patterns", IEEE Trans.Antennas Propaga., vol. AP-11, pp. 649-653, Jul. 1983. mode that generates the cross-polarization level is mainly the $\mathrm{TE}_{02}[11]$, which is also why the H-plane cross-polarization 